\title{
Deformation-mediated translocation of DNA origami nanoplates through a narrow solid-state nanopore
}

\author{
Libo Zhu, Zhen Zhang, Quanjun Liu*
}

State Key Laboratory of Bioelectronics, School of Biological Science and Medical

Engineering, Southeast University, No. 2, Sipailou, Nanjing 210096, China.

Corresponding author: lqj@seu.edu.cn

\section{Table of Content}

S1. Agarose gel electrophoresis of DNA origami nanoplates

S2. Original TEM images of purified DNA origami nanoplates

S3. Representative SEM images of nanopores.

S4. Raw continuous current traces for DNA nanoplates translocating through a $30 \mathrm{~nm}$ nanopore S6

S5. Raw continuous current traces for M13mp18 translocating through a nanopore $\ldots . . .$. S10

S6. The voltage dependence of current blockade of M13mp18 translocation ... S13

S7. Histograms of the current blockades of DNA nanoplate translocating through a $23 \mathrm{~nm}$ nanopore S14

S8. Raw continuous current traces for DNA nanoplates translocating through a $17 \mathrm{~nm}$ nanopore S15 


\section{S1. Agarose gel electrophoresis of DNA origami nanoplates}

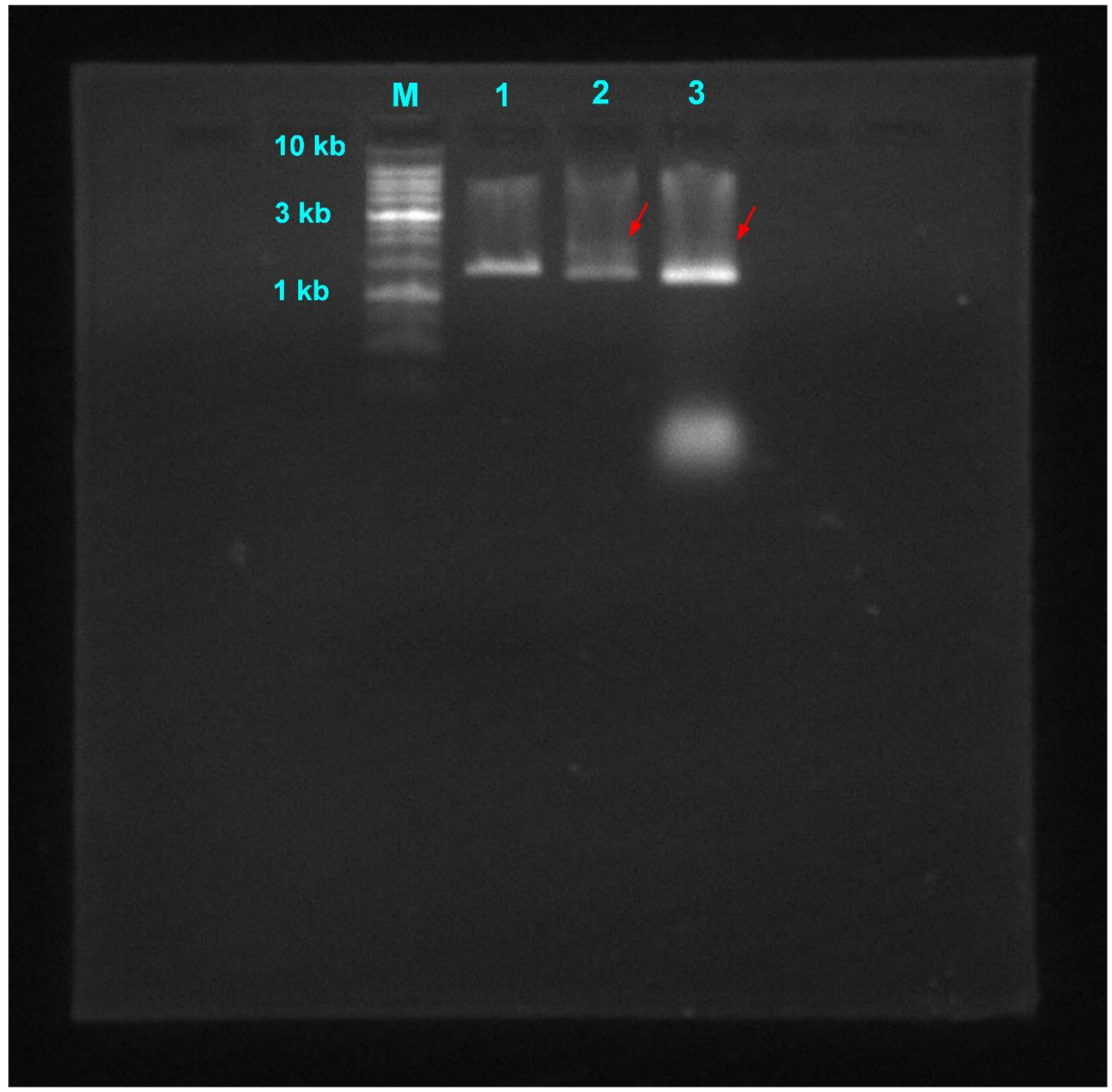

Figure S1. 1\% Agarose gel electrophoresis analysis of purified DNA origami nanoplates. The electrophoresis is performed in $11 \mathrm{mM} \mathrm{MgCl}_{2}$ solution, buffered with $0.5 \times \mathrm{TBE}$. Lane $\mathrm{M}: 1 \mathrm{~kb}$ DNA

Ladder; Lane 1: M13mp18 Scaffold strands; Lane 2: Purified DNA origami nanoplates; Lane 3: Unpurified DNA origami nanoplates. 


\section{S2. Original TEM images of purified DNA origami nanoplates}
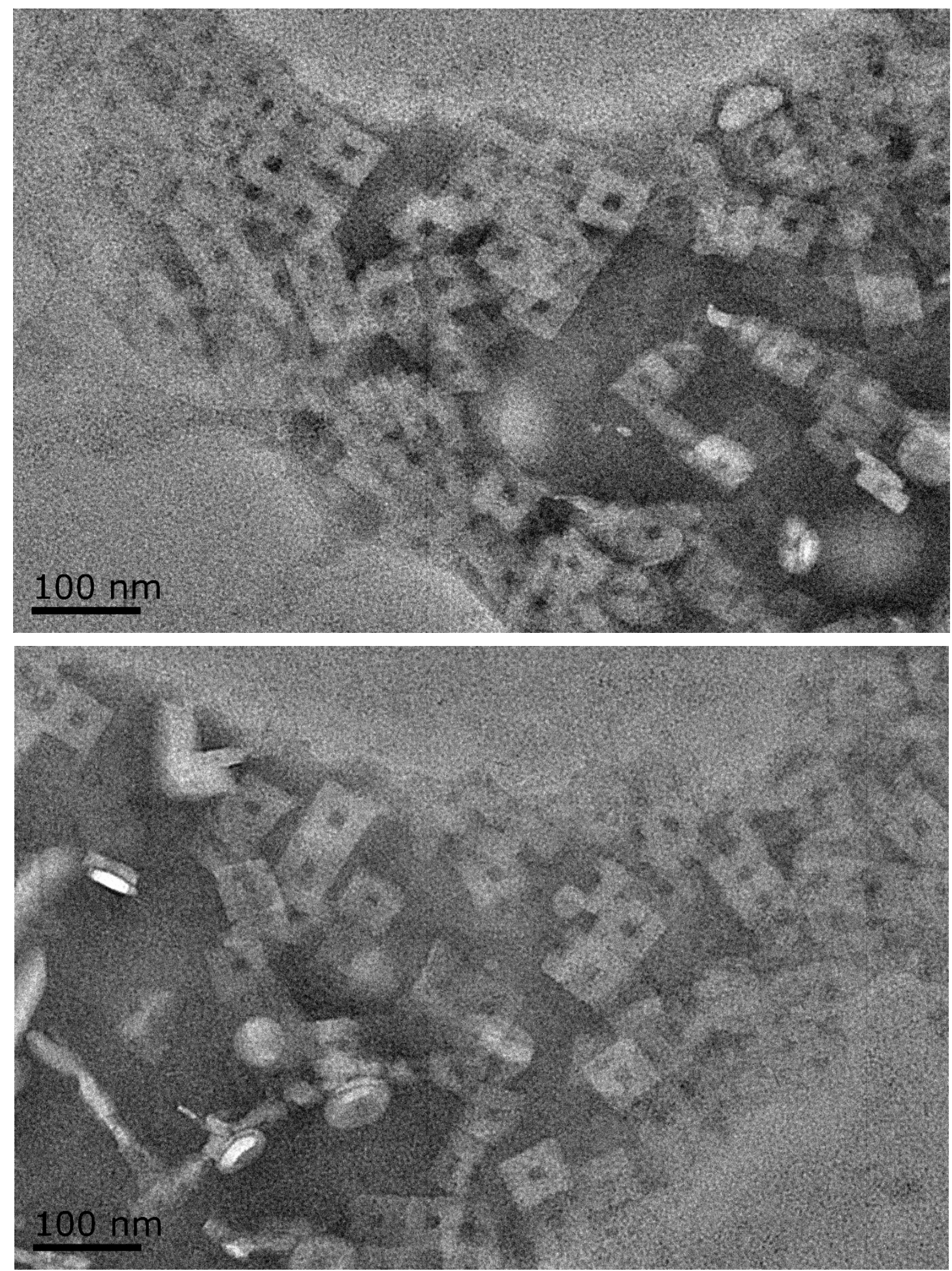

Figure S2. The original TEM images of DNA origami nanoplates. 


\section{S3. Representative SEM images of nanopores}

(a)

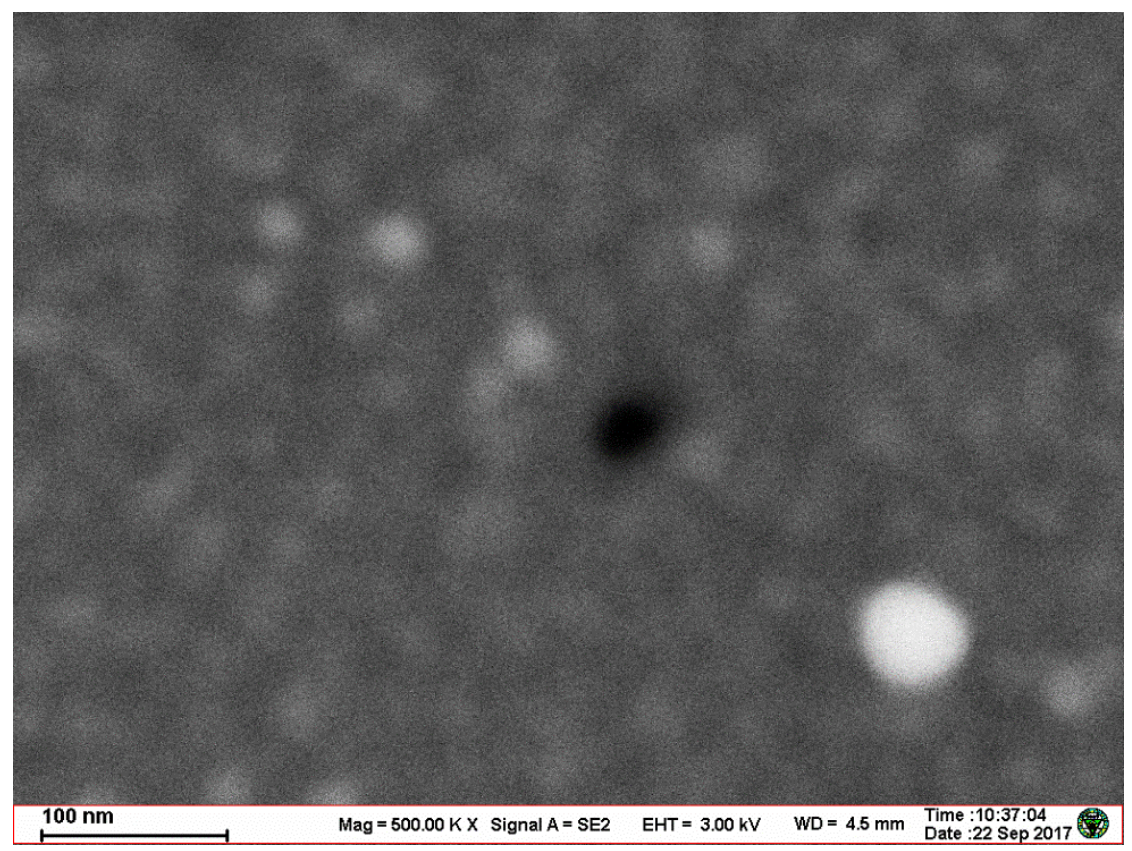

(b)

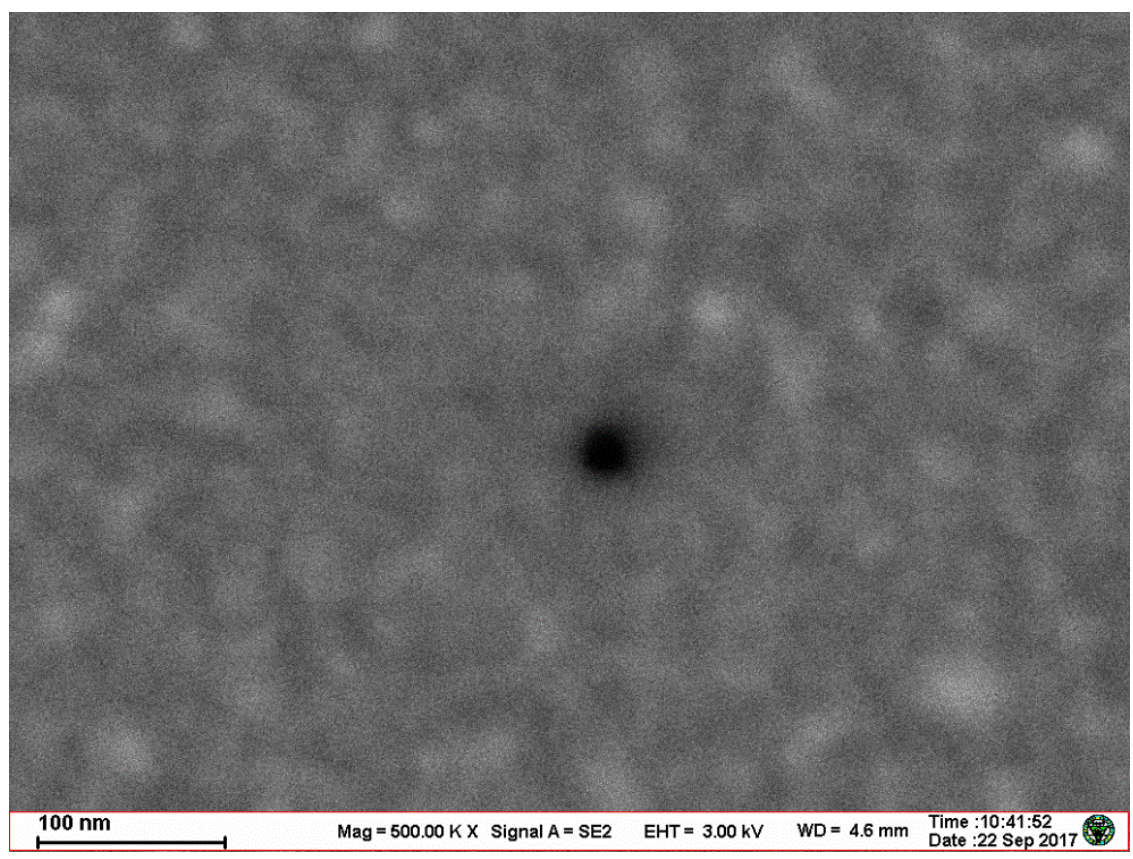


(c)

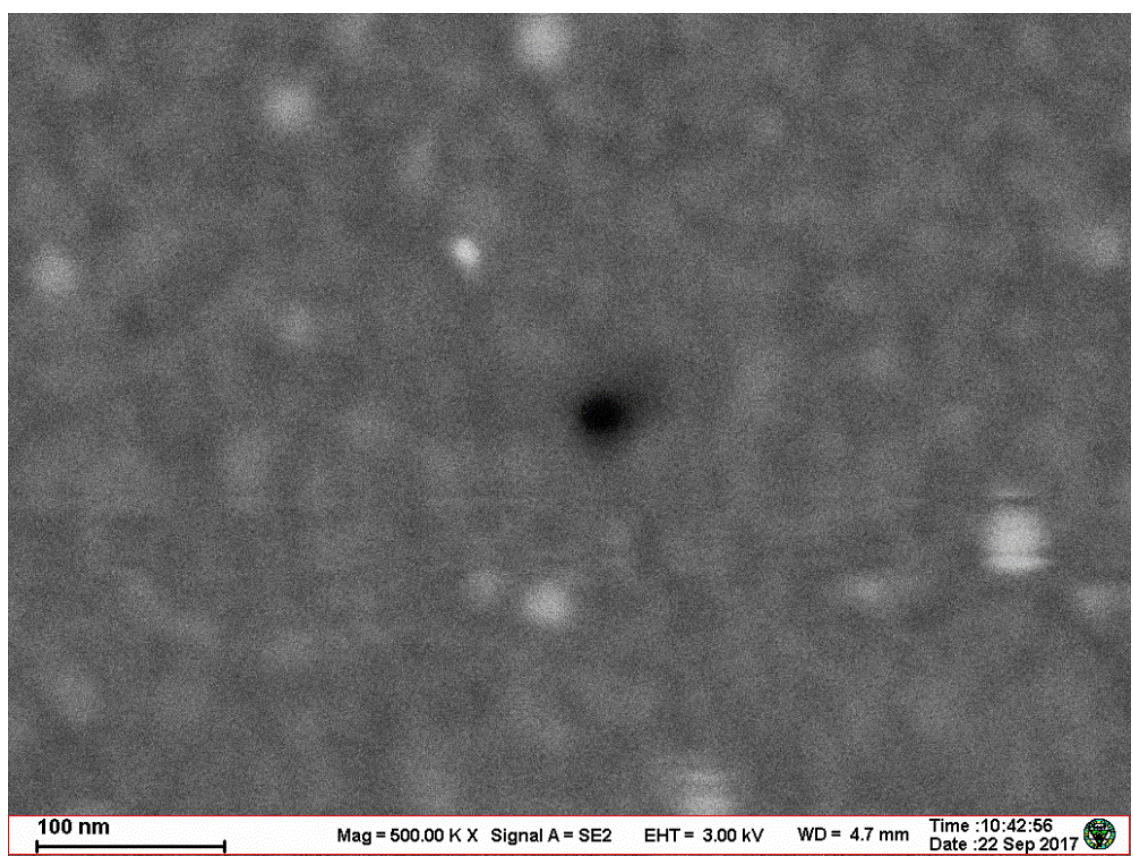

Figure S3. (a-c) Original SEM images of $30 \mathrm{~nm}$ diameter nanopores (a), $23 \mathrm{~nm}$ diameter nanopore (b) and $17 \mathrm{~nm}$ nanopore (c). 
S4. Raw continuous current traces for DNA nanoplates translocating through a 30

nm nanopore
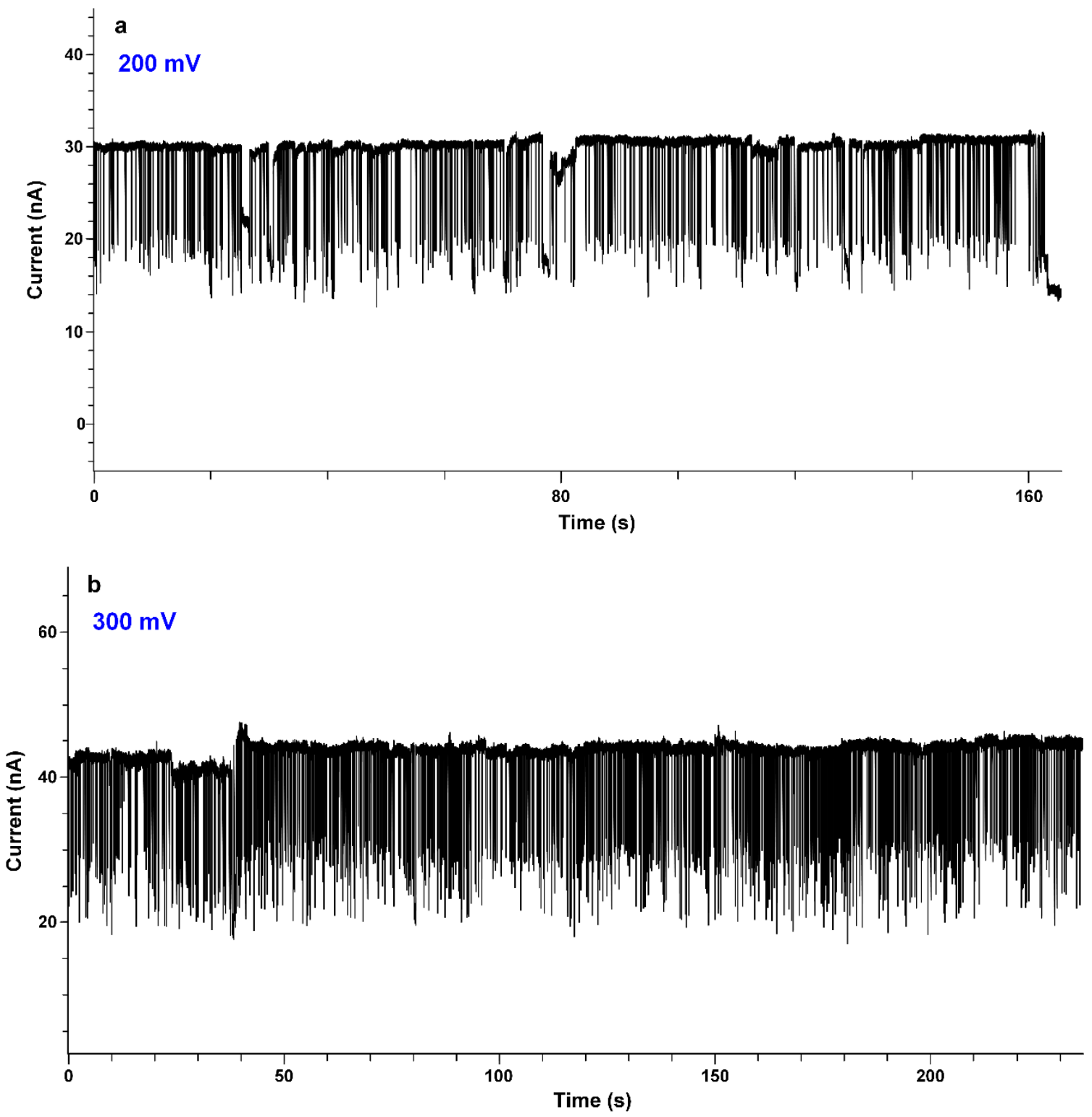

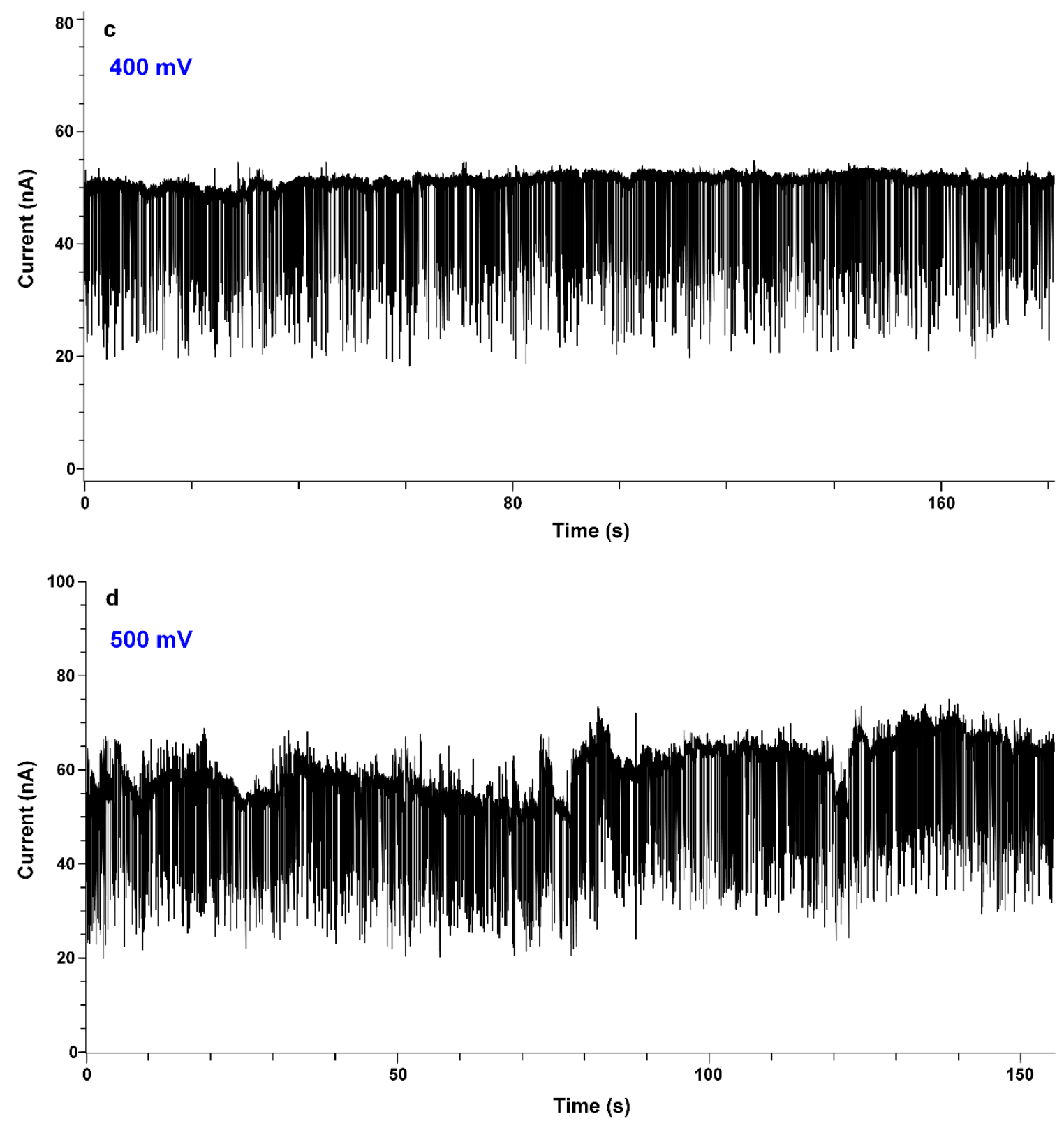

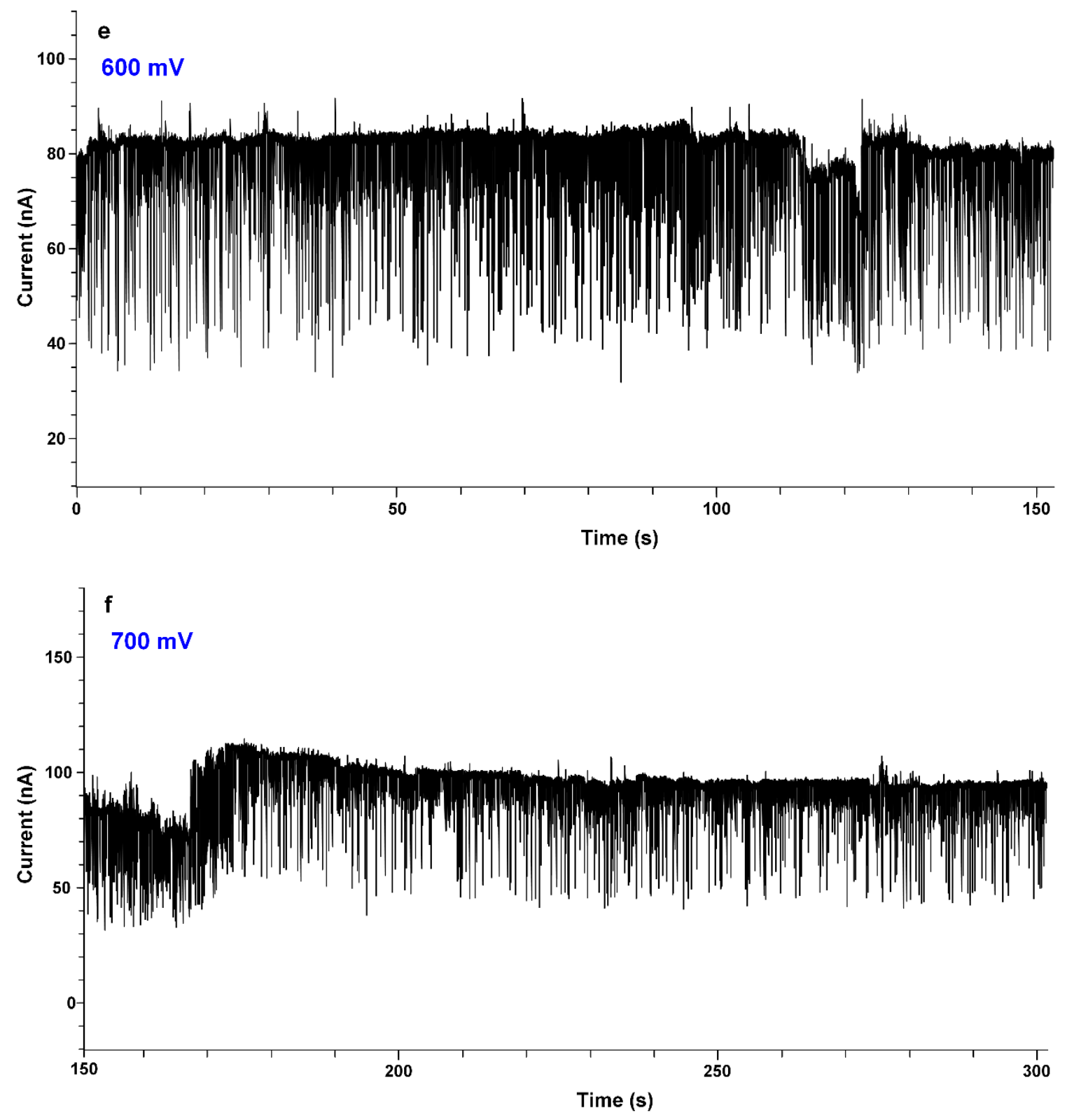


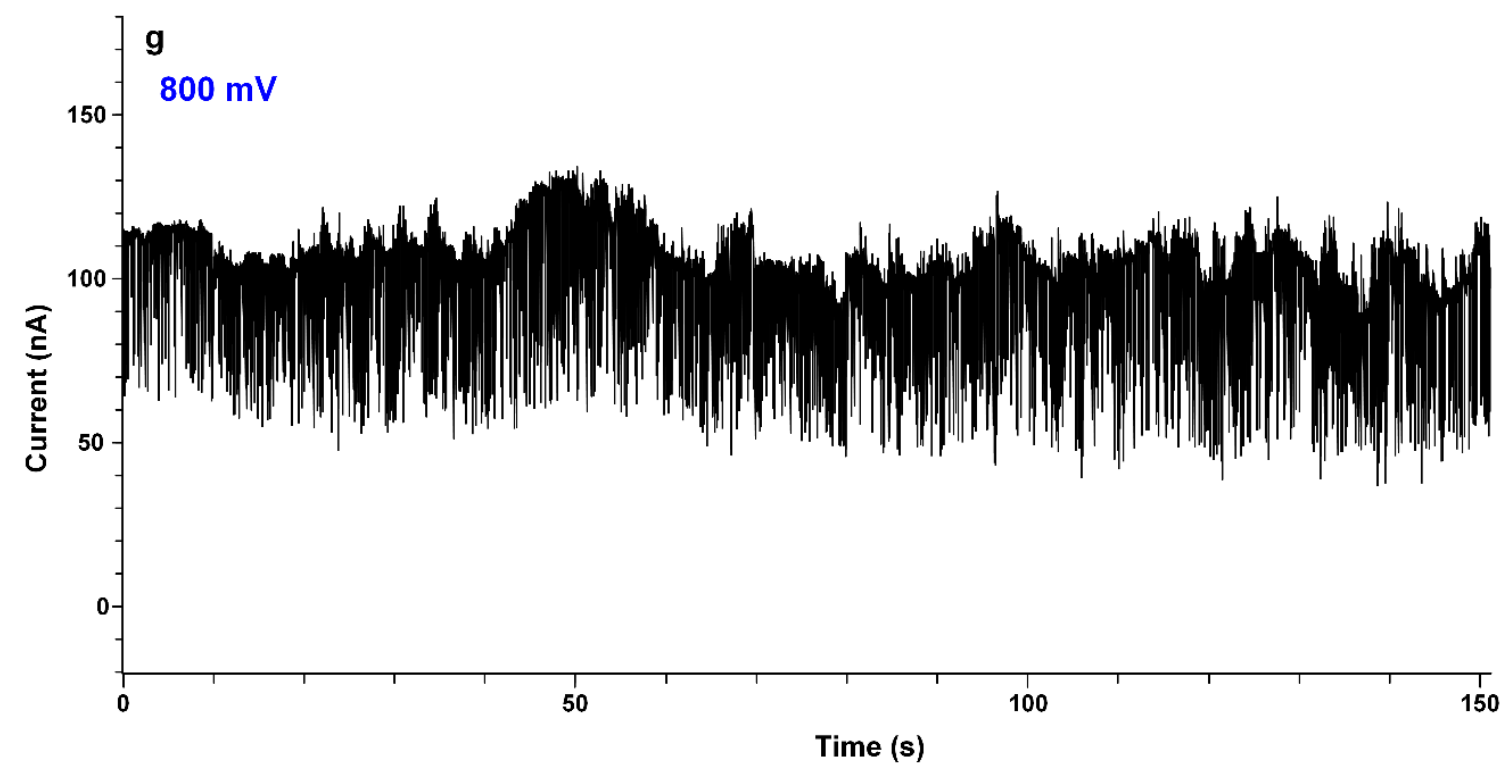

Figure S4. (a-g). Raw ionic current traces of DNA nanoplates translocating through a $30 \mathrm{~nm}$ in diameter nanopore under various voltages (200-800 mV). 
S5. Raw continuous current traces for M13mp18 translocating through a nanopore
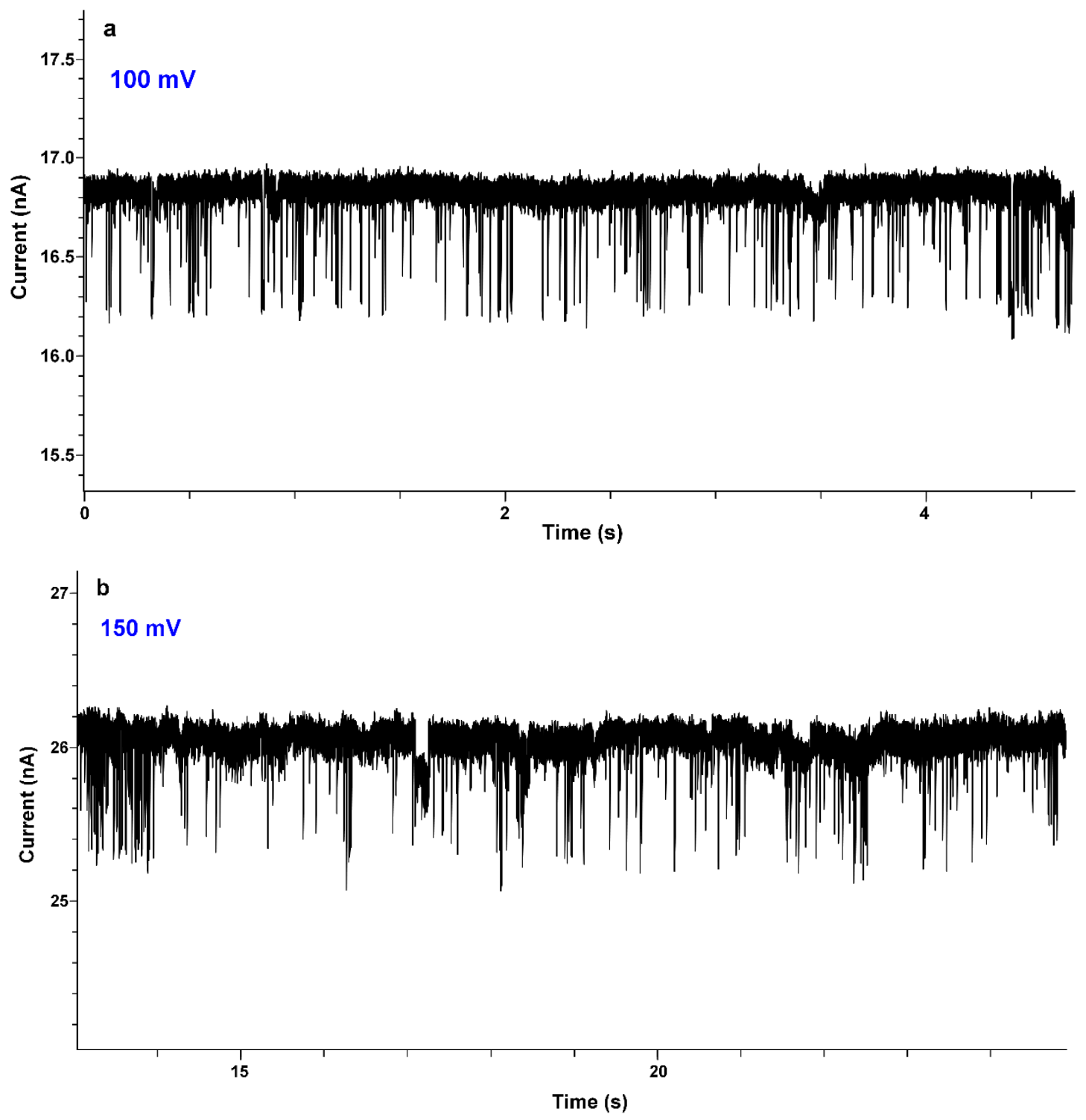

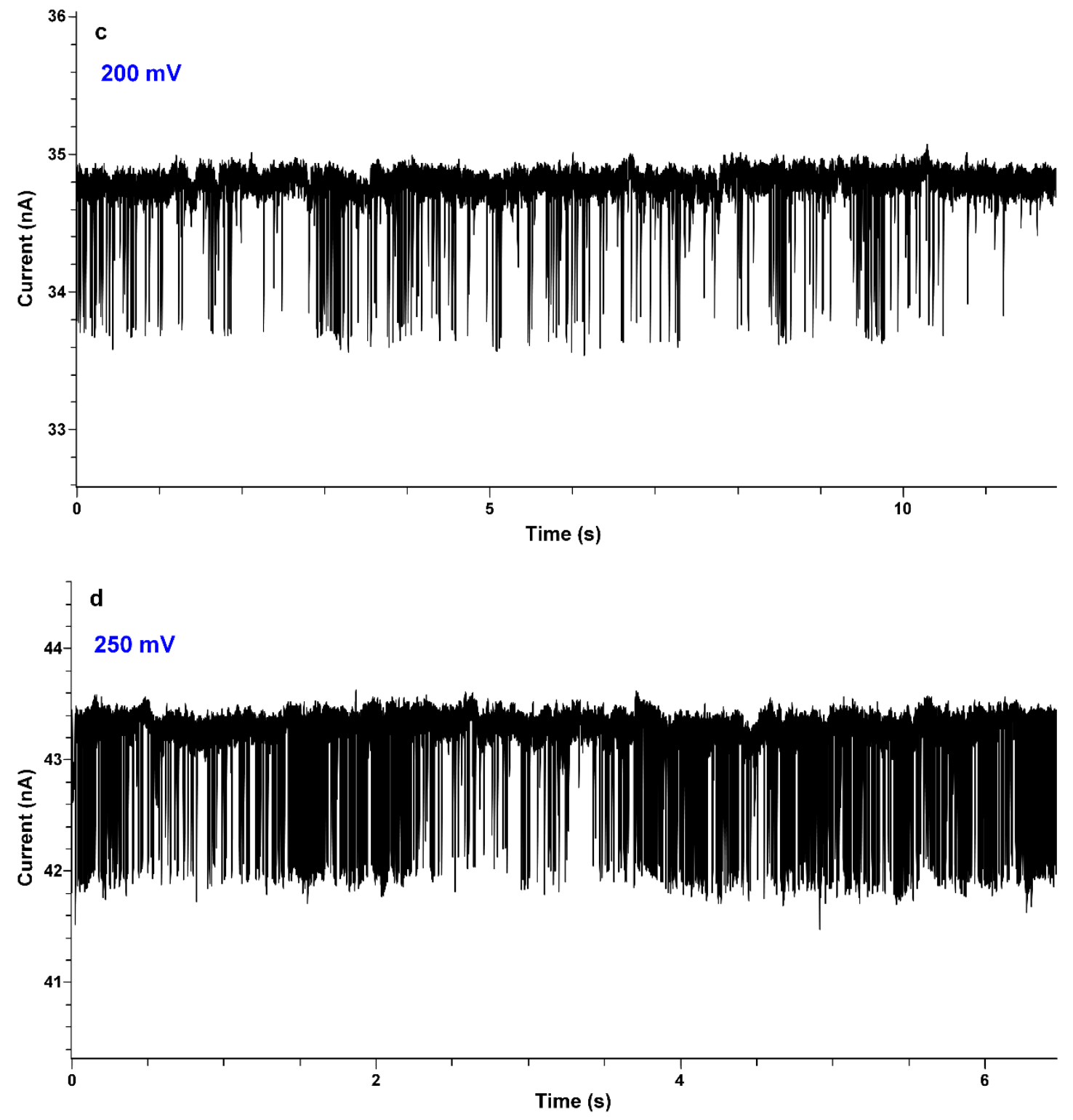


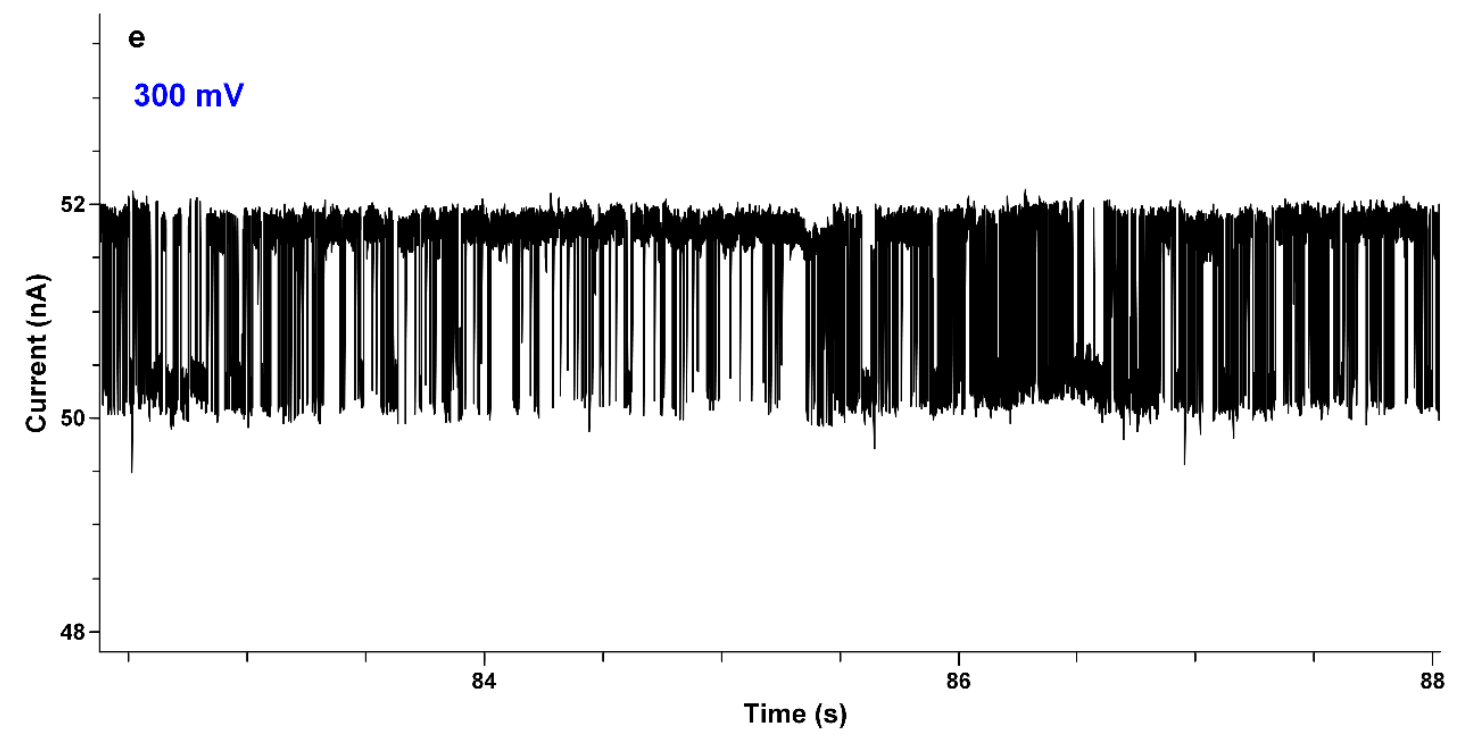

Figure S5. (a-e). Raw ionic current traces of M13mp18 translocating through a $\sim 27 \mathrm{~nm}$ in diameter nanopore under various voltages $(100-300 \mathrm{mV})$. 
S6. The voltage dependence of current blockade of M13mp18 translocation

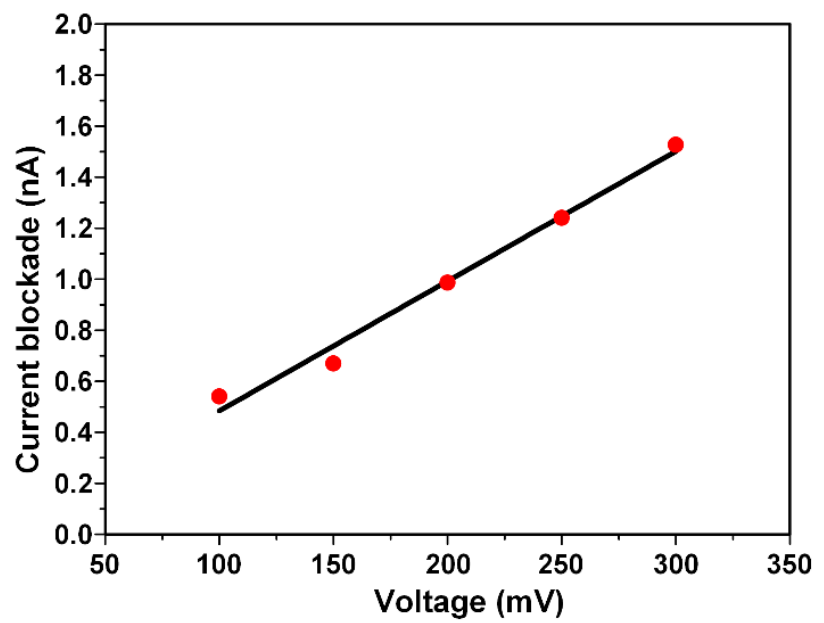

Figure S6. The current blockade of M13mp18 translocations as a function of biased voltage. 
S7. Histograms of the current blockades of DNA nanoplate translocating through a

\section{$23 \mathrm{~nm}$ nanopore}
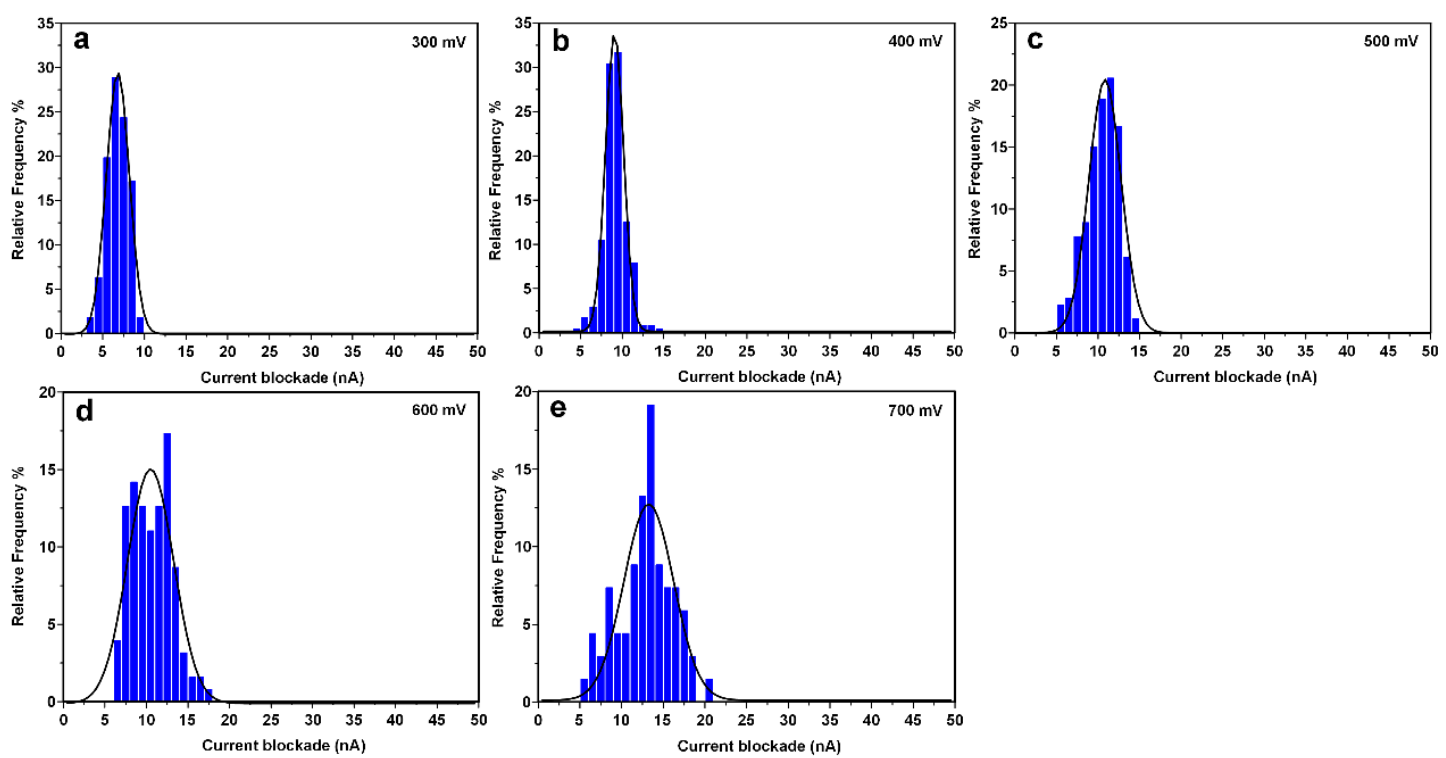

Figure S7. (a-e) Histograms of the current blockades resulted from DNA nanoplates transport through a $23 \mathrm{~nm}$ diameter nanopore at various voltages $(300-700 \mathrm{mV})$, the histograms are all fitted by Gauss. 
S8. Raw continuous current traces for DNA nanoplates translocating through a 17 nm nanopore
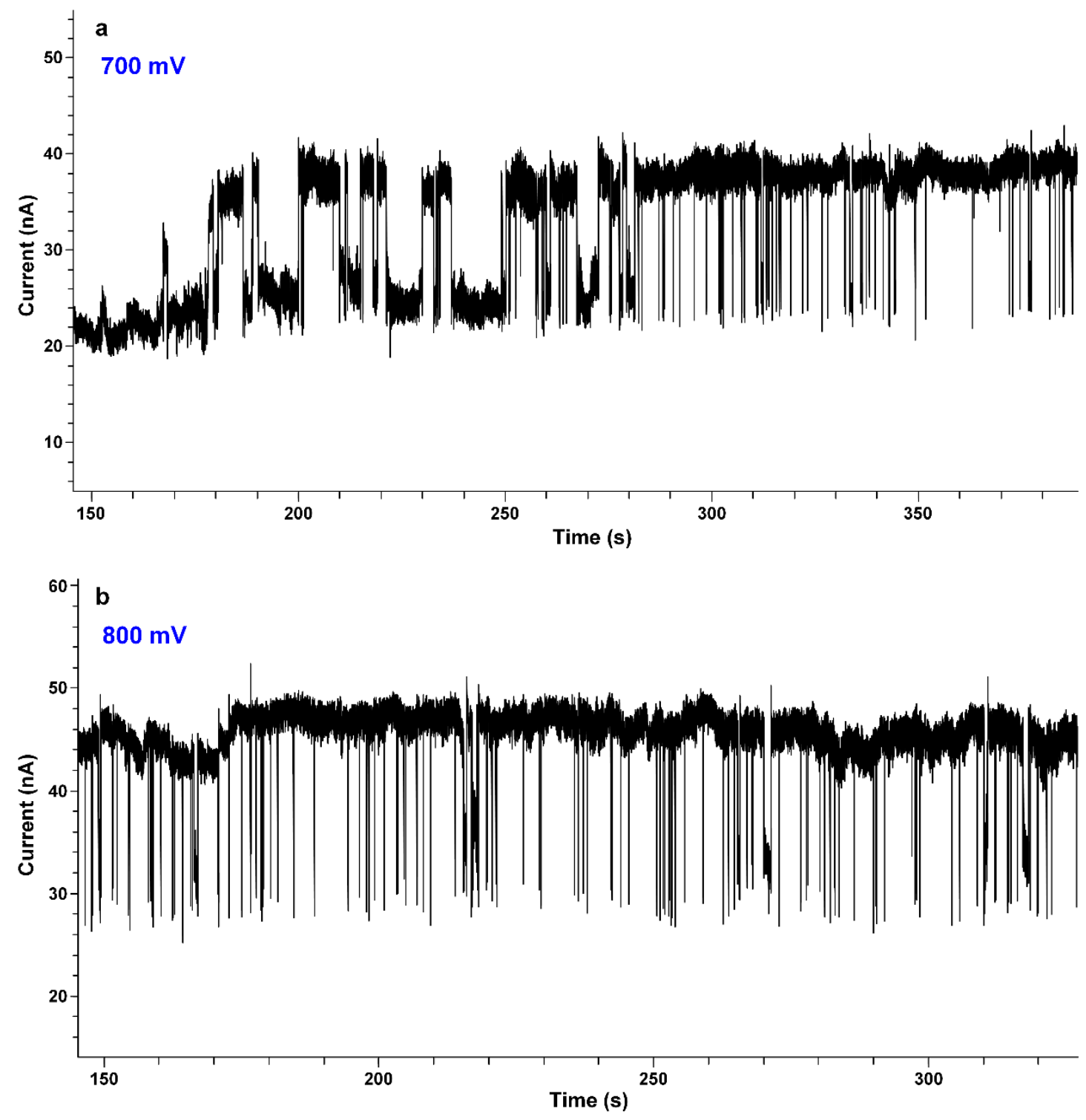


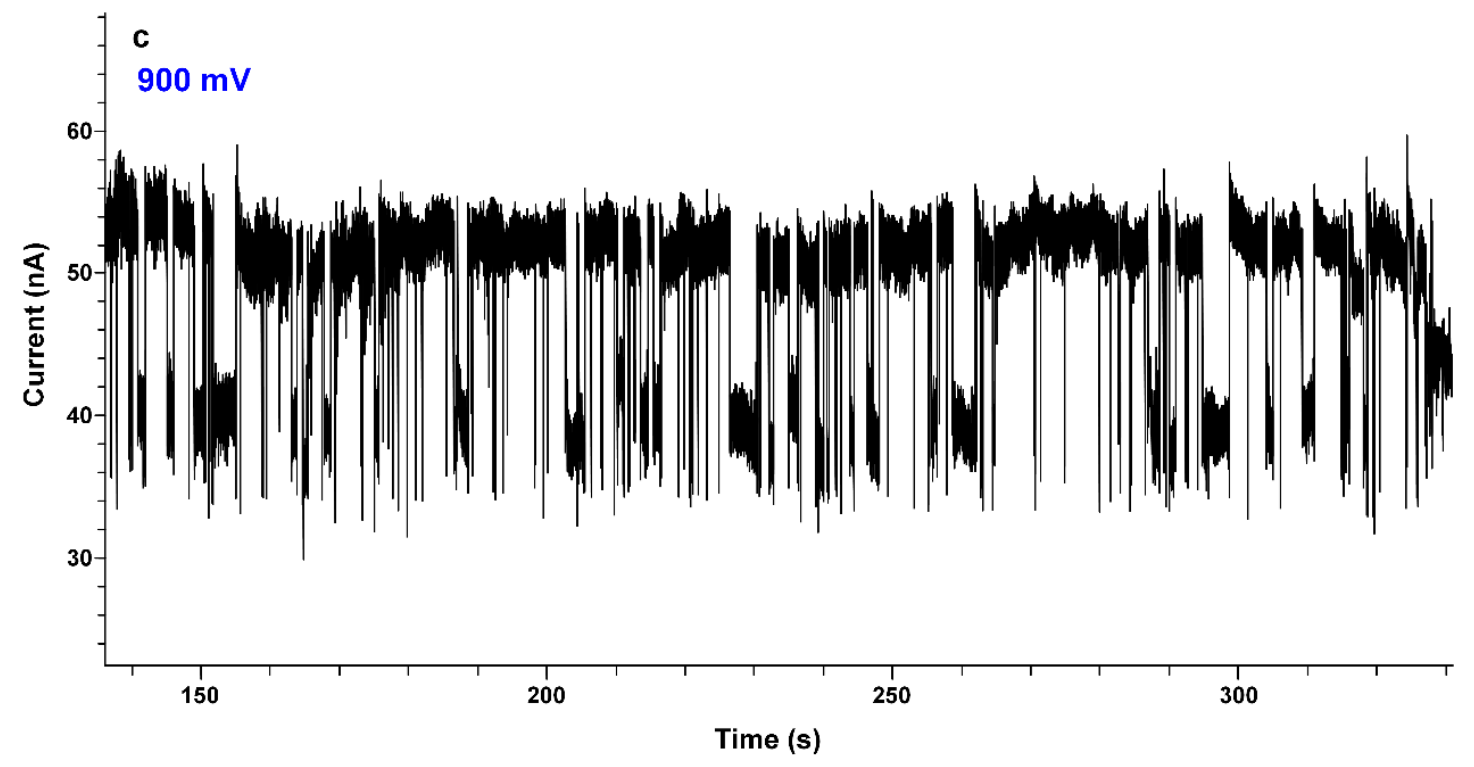

Figure S8. (a-c) Raw ionic current traces of DNA nanoplates translocating through a $17 \mathrm{~nm}$ diameter nanopore under various voltages $(700-900 \mathrm{mV})$. 